\title{
Clinical experiences of combining immunotherapy and radiation therapy in non-small cell lung cancer: lessons from melanoma
}

\author{
Anusha Kalbasi ${ }^{1}$, Ramesh Rengan ${ }^{2}$ \\ ${ }^{1}$ Department of Radiation Oncology, David Geffen School of Medicine, University of California Los Angeles, Los Angeles, CA, USA; ${ }^{2}$ Department \\ of Radiation Oncology, University of Washington School of Medicine, Seattle, WA, USA \\ Contributions: (I) Conception and design: All authors; (II) Administrative support: All authors; (III) Provision of study materials or patients: None; \\ (IV) Collection and assembly of data: A Kalbasi; (V) Data analysis and interpretation: All authors; (VI) Manuscript writing: All authors; (VII) Final \\ approval of manuscript: All authors. \\ Correspondence to: Ramesh Rengan, MD, PhD. Department of Radiation Oncology, 200 Suite B265 Medical Plaza Driveway, Seattle, WA, USA. \\ Email: rengan@uw.edu.
}

\begin{abstract}
Radiation therapy (RT) is an essential component of local control for non-small cell lung cancer (NSCLC), but distant failures dictate the poor prognosis of this disease. Until recently, the possibility of using RT as an immunoadjuvant to stimulate a systemic anti-tumor immune response was not a realistic clinical opportunity. The emergence of immune checkpoint blockade as an effective immunotherapy for NSCLC has opened the door for combinatorial approaches involving RT. In melanoma, the body of preclinical evidence combining radiation and immunotherapy buoyed clinical efforts, from which promising results have begun to emerge. Preclinical work combining radiation and immunotherapy indicate similar findings in NSCLC, and clinical efforts are ongoing. Here, we review the rationale, preclinical evidence, ongoing efforts and anticipated challenges of efforts combining radiation and immunotherapy in NSCLC.
\end{abstract}

Keywords: Radiation; immunotherapy; checkpoint blockade; non-small cell lung cancer (NSCLC); lung cancer

Submitted Oct 17, 2016. Accepted for publication Mar 14, 2017.

doi: $10.21037 /$ tlcr.2017.03.03

View this article at: http://dx.doi.org/10.21037/tlcr.2017.03.03

\section{Introduction}

The emergence of immunotherapy as the fourth pillar of cancer therapy, alongside surgery, radiation and chemotherapy, has generated interest in combinatorial strategies to maximize the benefits of anti-tumor immunity. The Food and Drug Administration (FDA) approval of immune checkpoint blockade in the treatment of metastatic non-small cell lung cancer (NSCLC) has specifically ignited efforts toward combinatorial approaches in NSCLC. Radiation therapy (RT) may be well suited as a partner to immunotherapy in NSCLC given its immunoadjuvant properties and its utility across the spectrum of patients with NSCLC. While combinations of RT and immunotherapy in NSCLC are still nascent, there is a body of preclinical literature supporting this approach. Moreover, emerging clinical data in melanoma and other disease sites support testing combinations of RT and immunotherapy in NSCLC.

\section{The need for greater systemic control in NSCLC}

NSCLC is the leading cause of cancer death in the United States with an estimated 158,080 deaths in 2016 (1). RT plays a prominent role in the treatment of NSCLC patients across the spectrum of disease: early stage, locally advanced and metastatic. In the growing population of patients with early stage disease, curative local therapy (surgery or stereotactic body radiotherapy) results in 5 -year survival rates below 55\% (SEER 2005-2011) and 3-year distant metastasis rates between $20-40 \%$ (2). For patients with locally advanced or metastatic disease, outcomes remain poor: 5 -year survival rates reach $25 \%$ for locally advanced stage IIIA patients (2). Patients with metastatic disease 
treated with platinum-doublet therapy have a median survival less than 1 year.

In the absence of better systemic disease control, even the most effective RT will have limited gains. Cytotoxic chemotherapy has had limited efficacy for patients with locally advanced and metastatic NSCLC. Several studies demonstrated a survival benefit to adjuvant chemotherapy after resection of non-metastatic NSCLC (stage II-III) (3-7). Adjuvant chemotherapy does not improve clinical outcomes in stage IA patients. For stage IB patients, CALGB 9633 found no benefit to adjuvant chemotherapy, notwithstanding a posthoc analysis suggesting benefit for patients with tumors $>4.0 \mathrm{~cm}(8)$. Even in the unplanned subgroup analysis of patients with tumors $>4.0 \mathrm{~cm}$, the modest benefit of adjuvant chemotherapy must be weighed against its toxicities.

Other approaches, including angiogenesis inhibitors, have had limited success $(9,10)$. The emergence of next generation sequencing has helped identify patients whose tumors harbor mutations for which targeted therapies exist. With this type of personalized medicine, profound responses are observed. However, this approach is limited to a small subset of patients whose tumors harbor actionable mutations (11).

Thus, additional approaches are needed to combat this devastating disease. Immunotherapeutic approaches are the most promising of the emerging therapies for NSCLC. These approaches were first successful in melanoma, a disease in which the immune activating factor IL-2 emerged as a standard therapy in the 1980s (12). Adoptive cell transfer of tumor infiltrating lymphocytes (TIL), and subsequently $\mathrm{T}$ cell receptor engineered lymphocytes, induced profound responses in melanoma in the 1990s and early 2000s. But it was not until 2011, when level I evidence demonstrated the survival benefit of immune checkpoint blockade targeting the immunoregulatory molecule CTLA-4, that immunotherapy attracted the attention of a global audience (13). This was followed closely by clinical trials demonstrating the efficacy of a second class of immune checkpoint inhibitors targeting programmed cell death protein 1 (PD-1) (14).

In 2015, immune checkpoint inhibitors targeting the PD-1 pathway were approved by the U.S. FDA for treatment of patients with locally advanced or metastatic NSCLC that had progressed on prior therapy. More recently the results of the KEYNOTE-024 trial randomized previously untreated patients with metastatic PD-L1 positive NSCLC to pembrolizumab, a humanized monoclonal antibody against PD-1, versus standard chemotherapy and showed a significant survival benefit in favor of patients receiving pembrolizumab (15).

\section{Rationale for immunotherapy in NSCLC: the shoe fits}

Before the approval of immune checkpoint blockade (antiPD1 therapy) for metastatic NSCLC, there were hints in studies of the immune microenvironment of these tumors that immunotherapy could be an effective approach. Several studies have examined the association of tumor infiltrating lymphocytes TIL on outcome in patients with NSCLC. The interest in TIL may be largely based on observations of heavy T-cell infiltrates in melanoma, a disease highly responsive to immunotherapy. In melanoma, the rich T-cell infiltrate serves as the basis for TIL therapy, in which TIL are grown ex vivo for therapeutic intravenous administration in combination with myeloablative regimens and immune growth factors (e.g., IL-2).

Across numerous studies, the presence of CD8+ T cells within the NSCLC tumor microenvironment was associated with superior clinical outcome, despite heterogeneous clinical populations and quantification methods (16). Even more recently, a study examined the association of TIL with survival in a more homogeneous population of patients with resected NSCLC who were enrolled on clinical trials testing adjuvant chemotherapy. The presence of TIL was associated with improved overall survival in this dataset (17). These data supporting TIL as a prognostic factor lend credence to the potential anti-tumor role of infiltrating $\mathrm{T}$ cells in NSCLC, as in melanoma.

In addition to TIL, the burden of somatic mutations in a particular tumor may determine its immunogenicity. Effective immunotherapy is based on the premise that $T$ cells recognize a non-self antigen presented by tumor cells. One hypothesis is that tumors with greater mutational load also have greater neoantigens repertoire, increasing the likelihood of a productive anti-tumor $\mathrm{T}$ cell response. Compared to other cancer types, melanoma has the highest burden of somatic mutations, thought to be a result of UV-light exposure (18). Consistent with this hypothesis, melanoma patients with higher mutational load are more likely to derive clinical benefit from anti-CTLA-4 immune checkpoint blockade (19).

The two major subtypes of NSCLC, squamous cell carcinoma and adenocarcinoma, carry the second and third highest mutational burden of all cancer types. This 
heavy mutational signature may be related to the effects of tobacco smoking. It is plausible that like melanoma, the greater mutational burden seen in NSCLC results in a higher likelihood of tumor reactive $\mathrm{T}$ cells that may mount a productive anti-tumor response under the appropriate stimulus. And in line with findings in melanoma, NSCLC patients with tumors harboring higher mutational load, antiPD-1 immune checkpoint blockade was more effective (20).

\section{Clinical data for immunotherapy in NSCLC}

The FDA approval of pembrolizumab was based on results of an international phase 1 study of pembrolizumab (KEYNOTE-001) that included 495 patients with locally advanced or metastatic NSCLC (including both squamous and non-squamous histology). In these patients there was an overall response rate of $19.4 \%$, including a response rate of $45.2 \%$ in the PD-L1 high-expressing population (21). Because of the latter finding, its approval was limited to patients with tumors expressing PD-L1.

Two trials compared an alternate antibody antagonist of PD-1, nivolumab, with docetaxel in the second-line treatment of metastatic squamous or non-squamous lung cancer (CheckMate-017 and CheckMate-057, respectively) $(22,23)$. For both CheckMate-017 and CheckMate-057, 2 -year overall survival was higher in the nivolumab arm ( $23 \%$ vs. $8 \%$ and $29 \%$ vs. $16 \%$, respectively). Unlike CheckMate-057, there was a statistically significant increase in progression-free survival for patients on CheckMate-017 and responses were not tied to baseline PD-L1 expression. Nivolumab was approved by the FDA in March of 2015 for treatment of advanced squamous cell NSCLC refractory to chemotherapy.

Despite the overwhelming success of immune checkpoint blockade, the majority of patients do not respond. Combination therapies-including those with RT-may circumvent the resistance mechanism and expand the efficacy of immune checkpoint blockade to larger fraction of patients with NSCLC.

\section{Rationale for radiotherapy as an immunoadjuvant}

Radiation is classically categorized as a DNA-damaging, cytotoxic therapy. However, in addition, it acts as an immunomodulator. The interest in radiation as an immunomodulator began nearly half a century ago, with the first anecdotal reports of the abscopal effect (24). The abscopal effect is a clinical response in a malignant lesion other than the target lesion (or a response outside the irradiated field), and was posited to be a result of an immunologic response incited by radiation to the target lesion.

Over the subsequent decades, scattered cases of the abscopal effect were reported, but these were met with healthy skepticism. As immune checkpoint blockade and other immunotherapies began to emerge over the past decade, reports of the abscopal effect became more prominent (25). Concurrently, the body of preclinical evidence on the intersection of radiation and immunity began to grow exponentially. These have been reviewed extensively elsewhere (26-28).

Importantly, the local effects of radiation were noted to be immune dependent in specific mouse models. In a murine model of melanoma, the depletion of CD8+ $\mathrm{T}$ cells abrogated the local effects of irradiation (29). Likewise, in a murine model of colon cancer, the local effects of radiotherapy were partially dependent on IFNalpha receptor and stimulator of interferon genes (STING) signaling (30). In both of these studies, the local anti-tumor effects of radiation could not be uncoupled from the $T$ cell immunity. That radiation can incite a local anti-tumor $\mathrm{T}$ cell response supports the possibility that it may incite a systemic anti-tumor $\mathrm{T}$ cell response, otherwise known as concomitant immunity (31).

Indeed, studies in an animal model of breast cancer demonstrated that ablative radiation of a local tumor impeded the development of lung metastases in a CD8+ T-cell dependent fashion (32). These results have been corroborated by several studies where local irradiation and systemic immunotherapy led to improved tumor control compared to either therapy alone (32-36).

Several mechanisms have been proposed by which radiation potentiates an anti-tumor $T$ cell response. Radiation can induce the release of danger signals or danger-associated molecular patterns (DAMPs), which serve as the first step in a cascade that leads to activation of antigen presenting cells (APCs)/dendritic cells. Danger signals, such as HMGB1, can bind to toll like receptors on the surface of APCs and potentiate the ability of APCs to activate nearby $\mathrm{T}$ cells (37). Thus, indirectly via release of DAMPs or via release of specific chemokines (e.g., GM-CSF), radiation may modulate antigen presentation. Radiation can also lead to increase in the quantity, variety and presentation of antigens from a tumor though activation of proteasome pathways, and augmentation of MHC class I presentation $(38,39)$. Radiation-induced chemokines may 
also directly attract APCs or effector $\mathrm{CD} 8+\mathrm{T}$ cells to the tumor microenvironment (40).

Radiation can also have immunosuppressive effects that may counteract the development of systemic antitumor immunity. Foremost, radiation can recruit immunosuppressive myeloid cells (e.g., myeloid derived suppressor cells, inflammatory monocytes, or tumorassociated macrophages) that either directly promote tumor outgrowth (41), or contribute to be an immunosuppressive microenvironment in which $\mathrm{T}$ cells are dysfunctional (42). Additionally, radiation has been shown in pre-clinical models to upregulate PD-L1 expression in the tumor microenvironment, which can lead to T-cell exhaustion (36). Finally, radiation can lead to accumulation of T-regulatory cells, which serve as obstacles to productive anti-tumor immunity (43).

Whether the dominant effects of radiation promote or disrupt anti-tumor immunity may largely depend on tumor type and context. In tumors with baseline immunogenicity, radiation may be more likely to stimulate productive anti-tumor immunity. On the contrary, immunologically "cold" tumors that lack a neoantigens signature and $\mathrm{T}$ cell infiltrate, may predominantly recruit immunosuppressive myeloid cells in response to radiation.

\section{Abscopal response to radiotherapy in NSCLC}

The majority of clinical data indicating an immunoadjuvant role for radiation are from patients with melanoma. However, data in NSCLC have also emerged. In 2013, clinicians reported a case of an abscopal response to radiotherapy in a patient with metastatic NSCLC who did not receive systemic therapy. The patient received conventionally fractionated radiation $(60 \mathrm{~Gy})$ for a T3N0 left upper lobe primary adenocarcinoma, and SBRT $(26 \mathrm{~Gy} \times 1)$ to a right lower lobe primary adenocarcinoma. Two weeks after treatment the patient was noted to have an FDG avid lesion in the adrenal gland consistent with metastasis, and approximately 2 months after treatment developed an FDG avid humeral lesion, also consistent with metastasis. One year after radiation, even without systemic therapy, these lesions had achieved complete metabolic response. The patient ultimately progressed at a different osseous site (44).

The potential for radiation to initiate a systemic antitumor immune response has been used as an argument for the treatment of early stage NSCLC with SBRT, especially in contrast to surgery. In a 2010 retrospective study comparing patients with T1-T2N0 NSCLC who underwent either wedge resection or SBRT, local and locoregional recurrence rates were lower in patients receiving SBRT (45). However, in the pooled analysis of two randomized trials of surgery versus SBRT in medically operable patients (STARS and ROSEL), while SBRT was as effective as surgery (46), there was no significant difference in the rate of regional recurrence or distant metastasis.

\section{Radiotherapy and immune checkpoint blockade}

The ability of radiation to initiate systemic anti-tumor immunity may be amplified in the context of immune checkpoint blockade. In animal models, radiation interacts favorably with immune checkpoint blockade. When combined with dual immune checkpoint blockade in a murine model of melanoma, radiation is associated with $\mathrm{T}$ cell receptor diversification, and results in greater control of non-irradiated tumors (47). A concordant phenomenon was seen in the peripheral blood from a cohort of patients with metastatic melanoma treated with immune checkpoint blockade (anti-CTLA-4) and palliative radiotherapy. In this cohort, $17 \%$ of patients experienced a response in the nonirradiated lesion, which exceeds the expected response rate for anti-CTLA-4 therapy alone.

In the largest dataset thus far examining the effects of local therapy in combination with immune checkpoint blockade (anti-CTLA-4), 127 metastatic melanoma patients were treated with electrochemotherapy, radiation or selective internal radiation therapy (SIRT). After accounting for measured differences in the treatment groups, patients receiving local therapy had significantly longer overall survival (48).

In NSCLC, there are case reports of abscopal responses to radiotherapy in patients also receiving immune checkpoint blockade. In one case, a patient with metastatic lung adenocarcinoma who had progressed despite multiple systemic therapy regimens, received RT concurrently with anti-CTLA-4 therapy and experienced a clinical response in multiple metastatic lesions (49). While it is possible this response was primarily related to anti-CTLA-4, it should be noted that anti-CTLA-4 therapy alone or in combination with systemic therapy has not resulted in improvements in disease outcomes for patients with NSCLC.

A recently reported phase I study of anti-CTLA-4 therapy with SBRT in patients with non-melanoma solid tumors included eight patients with NSCLC (50). Uniquely in this trial, patients received ablative doses of radiation, with BED of $\sim 100$ Gy (assuming alpha/beta $=10$ ) to the 
lung or liver. One out of eight NSCLC patients experienced either a partial response or prolonged (6 months) stable disease outside of the irradiated field (according to immune related response criteria).

\section{Toxicity considerations}

Multiple early phase studies have been conducted examining the safety of combining ablative radiotherapy with immunotherapeutic agents $(47,51,52)$. At the University of Pennsylvania, combination ipilimumab with hypofractionated palliative radiotherapy was tested in a phase I study. Of 21 enrolled patients, there was no grade 4 or higher toxicities. The most common grade 3 toxicity was anemia, which was unlikely to be related to the effects of focal hypofractionated radiotherapy.

For the combination of lung radiation and immune checkpoint blockade, pneumonitis is an overlapping toxicity. Radiation pneumonitis and pneumonitis due to immune checkpoint blockade may have shared mechanisms. The precise mechanism for radiation pneumonitis is unclear, but in addition to innate immune responses driven by monocyte, macrophage and neutrophil infiltration $(53,54)$, $\mathrm{T}$ cell driven adaptive immune processes have been implicated (55-57). The mechanism for pneumonitis related to immune checkpoint blockade has not been well studied, but is putatively due to autoreactive $\mathrm{T}$ cells.

Anti-PD-1 agents have a better safety profile than anti-CTLA4 agents. The rate of grade 3 toxicity for pembrolizumab in NSCLC in KEYNOTE-001 was $<10 \%$, including a $1.8 \%$ rate of grade 3 pneumonitis. And in modern SBRT series, the rate of grade 3 radiation pneumonitis is $<5 \%$ (58).

Thus, given the low independent rates of pneumonitis with each therapy, this combination will likely be safe and tolerable. However, given potential overlapping immune mechanisms of pneumonitis related to each therapy, it is important to carefully characterize the safety and toxicity profile in a prospective study. In the aforementioned phase I study of anti-CTLA-4 therapy with SBRT in patients with non-melanoma solid tumors, which included eight patients with NSCLC, clinical pneumonitis was not observed. A phase I safety study of atezolizumab (anti-PD-L1 antibody) in combination with SBRT in early stage NSCLC is actively recruiting patients (NCT02599454, www.clinicaltrials.gov). Likewise, in the metastatic setting, escalating doses of radiotherapy to the lung will be investigated combination with pembrolizumab to assess safety and toxicity
(NCT02587455).

\section{Ongoing studies}

In addition to the phase I study of SBRT combined with anti-PD-L1 in early stage NSCLC, other studies are examining combinations of immunotherapy and radiation or chemoradiation in NSCLC (Table 1). Many of the trials are conducted in the metastatic or oligometastatic setting, similar to those described above, wherein patients with NSCLC are included with other histologies and the emphasis is to examine the impact of SBRT on efficacy of immune checkpoint blockade or other immunotherapy. At one institution, investigators are testing FLT3 ligand, which may enhance antigen presentation, administered subcutaneously concurrent with SBRT in patients with metastatic NSCLC refractory to standard therapy (NCT02839265).

In studies of early stage and locally advanced disease, the focus of combination studies is to test the efficacy of immunotherapy when added to standard therapy. This includes immunotherapy other than immune checkpoint blockade, such as cancer vaccines that have been tested in the metastatic setting (e.g., cancer-testis antigens such as NY-ESO-1 and MAGE-A3, as well as telomerase and MUC-1). A phase II study of telomerase peptide vaccination in locally-advanced stage IIIA NSCLC suggested patients developed specific immune responses against the peptide in $80 \%$ of patients (59). Another vaccine study in locally advanced patients, this one a phase III randomized study, examined the impact of a vaccine against MUC1 glycoprotein on overall survival. While no overall survival difference was noted, the vaccine was associated with a benefit in a subset of patients who had received prior chemoradiotherapy (60).

Other vaccine studies are ongoing for patients after chemoradiation for locally advanced disease. However, combinations using immune checkpoint blockade have generated the most interest, and several trials are ongoing for locally advanced patients. RTOG will be studying adjuvant nivolumab after definitive chemoradiation for locally advanced NSCLC in a randomized phase III design (NCT02768558). Another institution is testing the use of neoadjuvant pembrolizumab for patients with stage IB, II or IIIA NSCLC in a single arm phase II design (NCT02818920). Durvalumab, an anti-PD-L1 monoclonal antibody, will be tested as a neoadjuvant therapy in combination with chemotherapy in patients with resectable stage IIIA NSCLC 


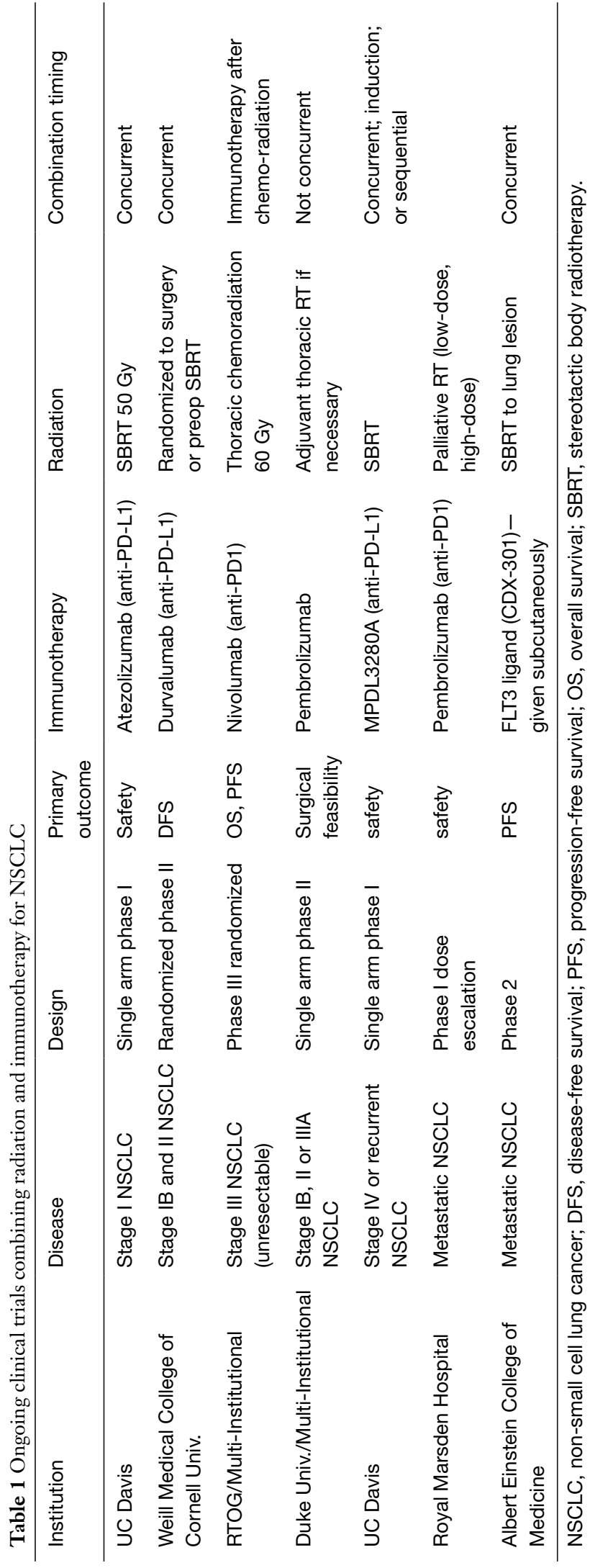

(NCT02572843). Patients will also receive adjuvant durvalumab, but only after adjuvant radiotherapy, should it be indicated. While these studies may prove a benefit for the experimental agent, they are unlikely to produce data on the potential synergy and radiotherapy.

\section{Resistance mechanisms/future directions}

While optimism is abounding regarding these combinatorial successes, failure of these approaches can and should be anticipated. In melanoma, where immune checkpoint blockade has had startling success, a substantial number of refractory cases remain. Some patients do not respond to therapy, and others respond and then progress. Recent evidence suggests that defects in interferon sensitivity within tumor cells may limit the efficacy of immune checkpoint blockade $(61,62)$. Interferon pathway stimulation is likely an important component of the radiotherapy-induced antitumor response as well (30). Thus, identifying patients with NSCLC that lack sufficient interferon pathway signaling, will allow for better selection of patients for clinical trials of combined radiation and immunotherapy.

\section{Conclusions}

Some of the immune effects of radiation can induce or boost systemic anti-tumor immunity, especially in tumor with baseline immunogenicity. By doing so, radiation can complement immunotherapies such as immune checkpoint blockade. This is an especially promising approach in NSCLC because of its shared features with melanoma, the disease where immunotherapy has made the most headway. Like melanoma, NSCLC is characterized by a significant number of somatic mutations and a substantial $\mathrm{T}$ cell infiltrate. Given these similarities, it is not surprising that NSCLC is one of a handful of malignancies that, like melanoma, responds to immune checkpoint blockade. With the emerging data in melanoma that radiotherapy may improve clinical response rates and outcomes with immune checkpoint blockade, this approach is equally intriguing in NSCLC. We await results from numerous studies testing this approach in the early stage, locally advanced and metastatic settings.

\section{Acknowledgements}

None. 


\section{Footnote}

Conflicts of Interest: The authors have no conflicts of interest to declare.

\section{References}

1. Ryerson AB, Eheman CR, Altekruse SF, et al. Annual Report to the Nation on the Status of Cancer, 1975-2012, featuring the increasing incidence of liver cancer. Cancer 2016;122:1312-37.

2. Howlader N, Noone A, Krapcho M, et al. SEER Cancer Statistics Review, 1975-2010. National Cancer Institute. Bethesda, MD. 2013. Available online: http://seer.cancer. gov/csr/1975_2010/

3. Arriagada R, Bergman B, Dunant A, et al. Cisplatinbased adjuvant chemotherapy in patients with completely resected non-small-cell lung cancer. N Engl J Med 2004;350:351-60.

4. Winton T, Livingston R, Johnson D, et al. Vinorelbine plus cisplatin vs. observation in resected non-small-cell lung cancer. N Engl J Med 2005;352:2589-97.

5. Douillard JY, Rosell R, De Lena M, et al. Adjuvant vinorelbine plus cisplatin versus observation in patients with completely resected stage IB-IIIA non-small-cell lung cancer (Adjuvant Navelbine International Trialist Association [ANITA]): a randomised controlled trial. Lancet Oncol 2006;7:719-27.

6. Pignon JP, Tribodet H, Scagliotti GV, et al. Lung adjuvant cisplatin evaluation: a pooled analysis by the LACE Collaborative Group. J Clin Oncol 2008;26:3552-9.

7. Butts CA, Ding K, Seymour L, et al. Randomized phase III trial of vinorelbine plus cisplatin compared with observation in completely resected stage IB and II nonsmall-cell lung cancer: updated survival analysis of JBR-10. J Clin Oncol 2010;28:29-34.

8. Strauss GM, Herndon JE, Maddaus MA, et al. Adjuvant paclitaxel plus carboplatin compared with observation in stage IB non-small-cell lung cancer: CALGB 9633 with the cancer and leukemia group B, radiation therapy oncology group, and North Central cancer treatment group study groups. J Clin Oncol 2008;26:5043-51.

9. Sandler A, Gray R, Perry MC, et al. Paclitaxel-carboplatin alone or with bevacizumab for non-small-cell lung cancer. N Engl J Med 2006;355:2542-50.

10. Patel JD, Hensing TA, Rademaker A, et al. Phase II study of pemetrexed and carboplatin plus bevacizumab with maintenance pemetrexed and bevacizumab as first-line therapy for nonsquamous non-small-cell lung cancer. J Clin Oncol 2009;27:3284-9.

11. Hirsch FR, Suda K, Wiens J, et al. New and emerging targeted treatments in advanced non-small-cell lung cancer. Lancet 2016;388:1012-24.

12. Rosenberg SA, Lotze MT, Yang JC, et al. Experience with the use of high-dose interleukin-2 in the treatment of 652 cancer patients. Ann Surg 1989;210:474-84; discussion 484-5.

13. Robert C, Thomas L, Bondarenko I, et al. Ipilimumab plus dacarbazine for previously untreated metastatic melanoma. N Engl J Med 2011;364:2517-26.

14. Topalian SL, Hodi FS, Brahmer JR, et al. Safety, activity, and immune correlates of anti-PD-1 antibody in cancer. $\mathrm{N}$ Engl J Med 2012;366:2443-54.

15. Reck M, Rodríguez-Abreu D, Robinson AG, et al. Pembrolizumab versus Chemotherapy for PD-L1Positive Non-Small-Cell Lung Cancer. N Engl J Med 2016;375:1823-33.

16. Bremnes RM, Busund LT, Kilvær TL, et al. The Role of Tumor-Infiltrating Lymphocytes in Development, Progression, and Prognosis of Non-Small Cell Lung Cancer. J Thorac Oncol 2016;11:789-800.

17. Brambilla E, Le Teuff G, Marguet S, et al. Prognostic Effect of Tumor Lymphocytic Infiltration in Resectable Non-Small-Cell Lung Cancer. J Clin Oncol 2016;34:1223-30.

18. Alexandrov LB, Nik-Zainal S, Wedge DC, et al. Signatures of mutational processes in human cancer. Nature 2013;500:415-21.

19. Snyder A, Makarov V, Merghoub T, et al. Genetic basis for clinical response to CTLA-4 blockade in melanoma. $\mathrm{N}$ Engl J Med 2014;371:2189-99.

20. Rizvi NA, Hellmann MD, Snyder A, et al. Cancer immunology. Mutational landscape determines sensitivity to PD-1 blockade in non-small cell lung cancer. Science 2015;348:124-8.

21. Garon EB, Rizvi NA, Hui R, et al. Pembrolizumab for the treatment of non-small-cell lung cancer. N Engl J Med 2015;372:2018-28.

22. Brahmer J, Reckamp KL, Baas P, et al. Nivolumab versus Docetaxel in Advanced Squamous-Cell Non-Small-Cell Lung Cancer. N Engl J Med 2015;373:123-35.

23. Borghaei H, Paz-Ares L, Horn L, et al. Nivolumab versus Docetaxel in Advanced Nonsquamous Non-Small-Cell Lung Cancer. N Engl J Med 2015;373:1627-39.

24. Ehlers G, Fridman M. Abscopal effect of radiation in papillary adenocarcinoma. Br J Radiol 1973;46:220-2. 
25. Postow MA, Callahan MK, Barker CA, et al. Immunologic correlates of the abscopal effect in a patient with melanoma. N Engl J Med 2012;366:925-31.

26. Kalbasi A, June CH, Haas N, et al. Radiation and immunotherapy: a synergistic combination. J Clin Invest 2013;123:2756-63.

27. Demaria S, Golden EB, Formenti SC. Role of Local Radiation Therapy in Cancer Immunotherapy. JAMA Oncol 2015;1:1325-32.

28. Schaue D, McBride WH. Opportunities and challenges of radiotherapy for treating cancer. Nat Rev Clin Oncol 2015;12:527-40.

29. Lee Y, Auh SL, Wang Y, et al. Therapeutic effects of ablative radiation on local tumor require $\mathrm{CD} 8+\mathrm{T}$ cells: changing strategies for cancer treatment. Blood 2009;114:589-95.

30. Deng L, Liang H, Xu M, et al. STING-Dependent Cytosolic DNA Sensing Promotes Radiation-Induced Type I Interferon-Dependent Antitumor Immunity in Immunogenic Tumors. Immunity 2014;41:843-52.

31. North RJ, Kirstein DP. T-cell-mediated concomitant immunity to syngeneic tumors. I. Activated macrophages as the expressors of nonspecific immunity to unrelated tumors and bacterial parasites. J Exp Med 1977;145:275-92.

32. Demaria S, Kawashima N, Yang AM, et al. Immunemediated inhibition of metastases after treatment with local radiation and CTLA-4 blockade in a mouse model of breast cancer. Clin Cancer Res 2005;11:728-34.

33. Zeng J, See AP, Phallen J, et al. Anti-PD-1 blockade and stereotactic radiation produce long-term survival in mice with intracranial gliomas. Int J Radiat Oncol Biol Phys 2013;86:343-9.

34. Liang H, Deng L, Chmura S, et al. Radiation-induced equilibrium is a balance between tumor cell proliferation and T cell-mediated killing. J Immunol 2013;190:5874-81.

35. Sharabi AB, Nirschl CJ, Kochel CM, et al. Stereotactic Radiation Therapy Augments Antigen-Specific PD1-Mediated Antitumor Immune Responses via CrossPresentation of Tumor Antigen. Cancer Immunol Res 2015;3:345-55.

36. Deng L, Liang H, Burnette B, et al. Irradiation and antiPD-L1 treatment synergistically promote antitumor immunity in mice. J Clin Invest 2014;124:687-95.

37. Apetoh L, Ghiringhelli F, Tesniere A, et al. Toll-like receptor 4-dependent contribution of the immune system to anticancer chemotherapy and radiotherapy. Nat Med 2007;13:1050-9.

38. Walter S, Weinschenk T, Stenzl A, et al. Multipeptide immune response to cancer vaccine IMA901 after singledose cyclophosphamide associates with longer patient survival. Nat Med 2012;18):1254-61.

39. Reits EA, Hodge JW, Herberts CA, et al. Radiation modulates the peptide repertoire, enhances $\mathrm{MHC}$ class I expression, and induces successful antitumor immunotherapy. J Exp Med 2006;203:1259-71.

40. Matsumura S, Wang B, Kawashima N, et al. Radiationinduced CXCL16 release by breast cancer cells attracts effector T cells. J Immunol 2008;181:3099-107.

41. Kalbasi A, Komar C, Tooker GM, et al. Tumor-Derived CCL2 Mediates Resistance to Radiotherapy in Pancreatic Ductal Adenocarcinoma. Clin Cancer Res 2017;23:137-48.

42. Seifert L, Werba G, Tiwari S, et al. Radiation Therapy Induces Macrophages to Suppress T-Cell Responses Against Pancreatic Tumors in Mice. Gastroenterology 2016;150:1659-72.e5.

43. Schaue D, Xie MW, Ratikan JA, et al. Regulatory T cells in radiotherapeutic responses. Front Oncol 2012;2:90.

44. Siva S, Callahan J, MacManus MP, et al. Abscopal [corrected] effects after conventional and stereotactic lung irradiation of non-small-cell lung cancer. J Thorac Oncol 2013;8:e71-2.

45. Grills IS, Mangona VS, Welsh R, et al. Outcomes after stereotactic lung radiotherapy or wedge resection for stage I non-small-cell lung cancer. J Clin Oncol. 2010 Feb 20;28(6):928-35.

46. Chang JY, Senan S, Paul MA, et al. Stereotactic ablative radiotherapy versus lobectomy for operable stage I nonsmall-cell lung cancer: a pooled analysis of two randomised trials. Lancet Oncol 2015;16:630-7.

47. Twyman-Saint Victor C, Rech AJ, Maity A, et al. Radiation and dual checkpoint blockade activate non-redundant immune mechanisms in cancer. Nature 2015;520:373-7.

48. Theurich S, Rothschild SI, Hoffmann M, et al. Local Tumor Treatment in Combination with Systemic Ipilimumab Immunotherapy Prolongs Overall Survival in Patients with Advanced Malignant Melanoma. Cancer Immunol Res 2016;4:744-54.

49. Golden EB, Demaria S, Schiff PB, et al. An abscopal response to radiation and ipilimumab in a patient with metastatic non-small cell lung cancer. Cancer Immunol Res 2013;1:365-72.

50. Tang C, Welsh JW, de Groot P, et al. Ipilimumab with Stereotactic Ablative Radiation Therapy: Phase I Results and Immunologic Correlates from Peripheral T Cells. Clin Cancer Res 2017;23:1388-96.

51. Golden EB, Chhabra A, Chachoua A, et al. Local 
radiotherapy and granulocyte-macrophage colonystimulating factor to generate abscopal responses in patients with metastatic solid tumours: a proof-of-principle trial. Lancet Oncol 2015;16:795-803.

52. Gulley JL, Arlen PM, Bastian A, et al. Combining a recombinant cancer vaccine with standard definitive radiotherapy in patients with localized prostate cancer. Clin Cancer Res 2005;11:3353-62.

53. Chen Y, Williams J, Ding I, et al. Radiation pneumonitis and early circulatory cytokine markers. Semin Radiat Oncol 2002;12:26-33.

54. Miura G, Awaya H, Matsumoto T, et al. Does granulocyte colony-stimulating factor exacerbate radiation-induced acute lung injury in rats? Radiat Med 2000;18:227-32.

55. Roberts CM, Foulcher E, Zaunders JJ, et al. Radiation pneumonitis: a possible lymphocytemediated hypersensitivity reaction. Ann Intern Med 1993;118:696-700.

56. Nakayama Y, Makino S, Fukuda Y, et al. Activation of lavage lymphocytes in lung injuries caused by radiotherapy for lung cancer. Int J Radiat Oncol Biol Phys 1996;34:459-67.

57. McBride $\mathrm{WH}$, Vegesna $\mathrm{V}$. The role of T-cells in radiation

Cite this article as: Kalbasi A, Rengan R. Clinical experiences of combining immunotherapy and radiation therapy in nonsmall cell lung cancer: lessons from melanoma. Transl Lung Cancer Res 2017;6(2):169-177. doi: 10.21037/tlcr.2017.03.03 pneumonitis after bone marrow transplantation. Int J Radiat Biol 2000;76:517-21.

58. Zhao J, Ling L, Yorke ED, et al. Simple Clinical Factors Associated With Radiation-Induced Lung Toxicity After Stereotactic Body Radiation Therapy in Lung Cancer: A Pooled Analysis of 70 Studies. Int J Radiat Oncol 2014;90:S60.

59. Brunsvig PF, Kyte JA, Kersten C, et al. Telomerase peptide vaccination in NSCLC: a phase II trial in stage III patients vaccinated after chemoradiotherapy and an 8-year update on a phase I/II trial. Clin Cancer Res 2011;17:6847-57.

60. Butts C, Socinski MA, Mitchell PL, et al. Tecemotide (L-BLP25) versus placebo after chemoradiotherapy for stage III non-small-cell lung cancer (START): a randomised, double-blind, phase 3 trial. Lancet Oncol 2014;15:59-68.

61. Zaretsky JM, Garcia-Diaz A, Shin DS, et al. Mutations Associated with Acquired Resistance to PD-1 Blockade in Melanoma. N Engl J Med 2016;375:819-29.

62. Gao J, Shi LZ, Zhao H, et al. Loss of IFN- $\gamma$ Pathway Genes in Tumor Cells as a Mechanism of Resistance to Anti-CTLA-4 Therapy. Cell 2016;167:397-404.e9. 